\title{
APPLICATION OF MICROSOFT EXCEL SOLVER TECHNIQUES TO COMPUTE CLUSTER SIZE IN CELLULAR WIRELESS NETWORK
}

\author{
Abhijit Sen, Kwantlen Polytechnic University, abhijit.sen@kpu.ca
}

\begin{abstract}
Microsoft Excel is one of the most popular software programs that is used by individual and business to record, manipulate and analyze data. Microsoft Excel offers number of data analysis tools collectively called "what if analysis". Many business problems are solved using these tools. However, in Engineering and Computing Science these features of Excel are seldom used to solve appropriate problems. Instead most of the problems are solved using specific tools such as MATLAB or specific Computer programs. In cases where Microsoft Excel is used, only basic operations such as formula, mathematical functions and graphing or charting are utilized. In this paper author attempts to show with examples how Excel Solver techniques can effectively be used to model and solve common problems in Wireless Communication network. Many of the wireless network problems require extensive problem solving skills to manipulate mathematical expressions to produce solutions. This pedagogical approach of using Excel Solver in handling some difficult computation problem in Wireless Communication network is specially aimed at students who do not have strong mathematical aptitude in solving computational problems. A very common example of finding Cell clusters in Wireless Cellular network is used to demonstrate the usefulness of Excel Solver in modeling and solving complex optimization problems. The author also discusses the limitations and practical issues related to using Excel solver.
\end{abstract}

Keywords: cell clusters, cellular network, channel, frequency, signal-to-co-channel interference

\section{INTRODUCTION}

In Cellular network, a large geographical service area is divided into number of small areas - each small area is called a cell. The number of cells together form a cluster. Each individual cell in a cluster is allocated different frequency. Because of the limitation of available frequency bandwidth, cellular networks reuse the same frequency at different clusters. The cell cluster size in a Cellular Wireless Network is determined using well known algorithm (Mark et al., 2003) which is described in subsequent section. In this paper the author explores the possibility of using Excel Solver techniques to perform various wireless communication computational problems and demonstrates the practicality of using these techniques in the areas of wireless communication by solving one specific problem. The methodology can be used with other optimization problems in cellular network. These techniques can be easily applied by users without strong mathematical proficiency to device a solution.

We have presented an example of nonlinear optimization problems in Cellular Wireless Communication and showed how these problems can be solved easily using Excel Solver. The computational simplicity of Excel allows one to generate different example scenarios, to experiment by changing the various input parameters and to observe the outputs providing insight into the problem without going through complexities of analytical solutions. The objective of this paper is to show how one can use Excel Solver to implement the algorithm to determine the optimal cluster size in a cellular wireless network.

\section{LITERATURE SURVEY}

The use of spreadsheet modeling and Excel Solver in solving linear and nonlinear programming problems in an introductory Operations Research course is discussed in literatures (Chandrakantha, 2011; Winston, 2014). The internet has many examples and tutorials dealing with the application of Excel Solver for various applications areas such as manufacturing, transportation, financial planning, capital investment. However, one does not find many uses of Excel Solver to solve engineering or computer science problems. The fundamental concepts of optimization, and usage of Microsoft Excel techniques to specific application areas of business and marketing are detailed in (Winston, 
2014). In Engineering and Computer Science disciplines where Excel is applied to solve specific problems, one finds only the usage of rudimentary functionalities of Excel such as use of simple mathematical formulas, operations, and charting. The limitations of Excel for real life examples are discussed in (Evans, 2008). But these limitations may not affect the examples used to convey the theoretical understanding of the topic and methodology of the problem solution. This paper discusses one use of Excel Solver in solving a common computation in cellular wireless network. The rest of the paper is organized as per the following manners. The basic concept of Cellular System, and algorithm of finding cell cluster is discussed in "Overview of Cellular System" section. Modeling of the Cluster algorithm is discussed in "Excel Solver Modeling of Signal-to-Cochineal Interference Algorithm". Section titled "Determining Cluster Size Using Microsoft Excel Solver" discusses step by step procedures for implanting Excel Solver. The output of Excel Solver specifying the optimal cluster size is shown in section titled "Result". Limitations of implementation is discussed in section titled "Limitations of Excel Solver". followed by conclusion in section titled "Conclusions".

\section{OVERVIEW OF CELLULAR SYSTEM}

In cellular systems, the total service area is divided into a number of smaller areas, each powered by small transmitter, defined as "cell" as shown in Figure 1. Each cell provides coverage only to a small area. The total coverage area is divided into clusters denoted by $\mathrm{N}$. The same groups of channels may be used to cover different cells that are separated from one another by distances large enough to keep the interference levels within tolerable limits. There can be no cochannel interference within a cluster. This is because neighboring cells do not use same set of frequencies to prevent interference. The theoretical concepts on Cellular networks are extensively discussed in literatures (Stalling, 2016; Stubert, 2017).

Any regular shaped polygons may be used to represent the cell; most popular cell geometry is hexagonal shape. Cells which use the same frequency are called "co-channel cells" (e.g., all cells labeled A or numeric 1, use the same frequency, all cells labelled B or numeric 2 use another frequency).

Cells with the same label use the same set of frequencies. Frequency re-use is limited by co-channel interference. Cochannel interference occurs when different cells simultaneously use the same frequency channel. To combat interference, number of steps can be taken such as power control effective allocation frequencies to name a few (Adediran, Lasisi, Okedere; 2017; Waterloo, 2012).

To find nearest co-channel neighbors in a hexagonal chain one can use the following steps (Figure 1):

- Move over i number of cells in any chain of hexagons

- Move over j number of cells after turning 60 degrees counter-clockwise where both $\mathrm{i}$ and $\mathrm{j}$ are non-negative integers.

For a hexagonal coverage area in Figure 1, cluster size N, can be calculated using appropriate values of $i$, and $j$ using the formula (1) as shown below.

Cluster size $\mathrm{N}$ must satisfy the following equation

$\mathrm{N}=\mathrm{i} * \mathrm{i}+\mathrm{i} * \mathrm{j}+\mathrm{j} * \mathrm{j}$, where $\mathrm{i}, \mathrm{j}=0,1,2,3 \ldots$

In Figure 1, with $\mathrm{i}=2, \mathrm{j}=1$ the above formula (1) gives $\mathrm{N}=7$. In other words, a cluster consists of cells $\mathrm{A}, \mathrm{B}, \mathrm{C}, \mathrm{D}, \mathrm{E}$, F, and G. Each cell in cluster A, B, C, D, E, F, and G must be assigned different frequencies to prevent interference. 


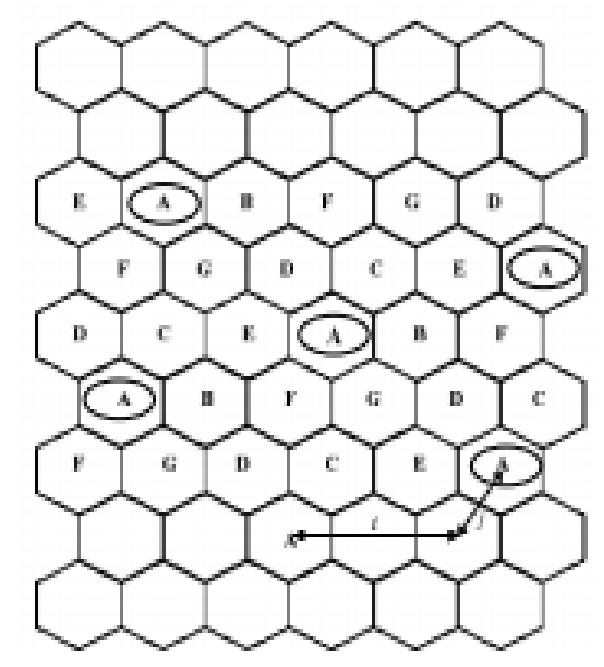

Figure 1. Frequency Reuse Pattern for Cluster Size $N=7$ (Source: https://ece.uwaterloo.ca/ ece414/ece414_chapter5_w12.pdf)

Table 1 shows the number of cells in a cluster based on different values of $i$ and $j$.

Table 1. Sample Cluster Size using formula (1) $N=i * i+i * j+j * j, i, j=0,1,2,3 \ldots$

\begin{tabular}{|c|c|c|}
\hline Values of $\mathbf{i}$ & Values of $\mathbf{j}$ & $\begin{array}{c}\text { Cluster Size } \mathbf{N} \\
\mathbf{N}=\mathbf{i} * \mathbf{i}+\mathbf{i} * \mathbf{j}+\mathbf{j}^{*} \mathbf{j}\end{array}$ \\
\hline & & 4 \\
\hline $\mathbf{2}$ & $\mathbf{0}$ & $\mathbf{4}$ \\
\hline $\mathbf{0}$ & $\mathbf{2}$ & $\mathbf{4}$ \\
\hline 2 & 2 & $\mathbf{1 2}$ \\
\hline
\end{tabular}

Figure 2 below shows possible configuration of clusters for different initial values of $i$, and $j$. Figure 2 (a) shows cluster consists of 4 cells 1, 2, 3, and 4, Figure2 (b) cluster consists of 7 cells 1, 2, 3, 4, 5, 6, and 7 and Figure 2 (c) shows cluster consists of 12 cells $1,2,3,4,5,6,7,8,9,10,11,12$. Each of these cells in a single cluster will be allocated different frequencies or channels. 


\section{Issues in Information Systems}

Volume 21, Issue 1, pp. 37-48, 2020

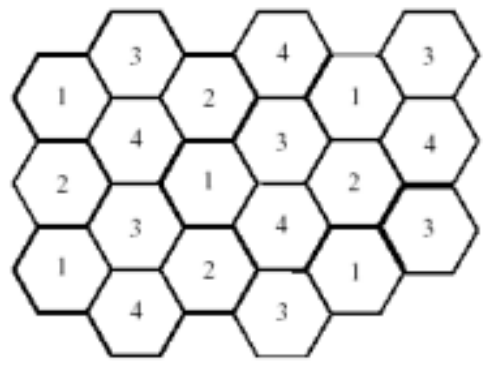

(a) $i=2$ and $j=0$
$N=4$

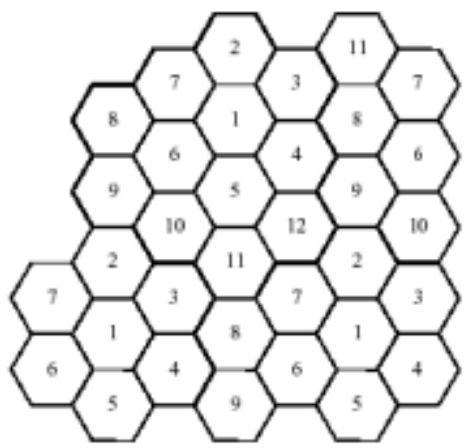

(c) $i=2$ and $i=2$

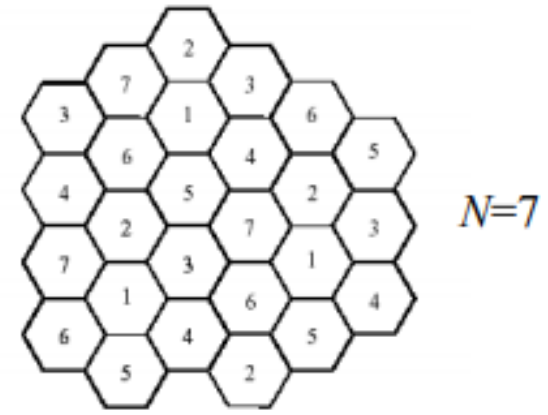

(b) $i=1$ and $j=2$

$N=12$

Figure 2. Sample Cluster Size with different values of $\mathrm{I}$, and $\mathrm{j}$

(Source: https://ece.uwaterloo.ca/ ece414/ece414_chapter5_w12.pdf)

As is seen from Figure 1 and Figure 2, in cellular system frequency channels are reused to serve number of users because of limited availability of bandwidth. One of the factors that limit reuse of frequency channels is co-channel interference (El-Jaafreh, 2000). Co-channel interference is produced by simultaneous use of the same frequency channel in different cells. The phenomenon of co-channel interference is investigated by number of researchers (Diakoku K et al.,1983; Cox, 1982; Safak et al., 1991). If the cluster size N is increased, the distance between nearest co cells increases, resulting in smaller co-channel interference. So frequency reuse must be carefully planned to keep co-channel interference at an acceptable level. The performance parameter that is used to decide cluster size is signalto-co-channel interference ratio (S/I), which is given by the following equation (Mark et al., 2003):

$\mathrm{S} / \mathrm{I}=1 /\left(2(\mathrm{q}-1)^{-\mathrm{k}}+2 \mathrm{q}^{-\mathrm{k}}+2(\mathrm{q}+1)^{-\mathrm{k}}\right)$

Where

$\mathrm{S}$ is the power of desired signal

$\mathrm{I}$ is the power of co-channel interference

$\mathrm{k}$ is called path loss exponent (Mark et al., 2003) and is normally determined by experiment. In many cases it is in the range

$2<=\mathrm{k}<=5$

$\mathrm{q}$ is frequency reuse ratio given by the following formula.

$\mathrm{q}=$ sqrt $(3 * \mathrm{~N})$ for hexagonal geometry (Mark et al., 2003)

$(\mathrm{S} / \mathrm{I})_{\mathrm{dB}}=10 \log _{10}(\mathrm{~S} / \mathrm{I})$

Typically cluster size $\mathrm{N}$ must be chosen such a way that $(\mathrm{S} / \mathrm{I}) \mathrm{dB}$ must be greater than $18_{\mathrm{dB}}$ for good reception (Mark et al., 2003). 


\section{EXCEL SOLVER MODELING OF SIGNAL-TO-CO-CHANNEL INTERFERENCE ALGORITHM}

Solver is part of what-if analysis tools in Microsoft Excel, and may be used to find maximum or minimum value for a formula in one cell - called the objective cell — subject to constraints, or limits, on the values of other formula cells on a worksheet. Solver works with a group of cells, known as decision variables or simply variable cells that participate in computing the formulas in the objective and constraint cells. Solver adjusts the values in the decision variable cells to satisfy the limits on constraint cells and produce the result you want for the objective cell.

In this section, the author models the signal-to-co-channel interference algorithm using Excel Solver. The relevant formulae used for implementation are outlined in the previous section.

Problem Statement: Find the cluster size N when the value of signal-to-co-channel interference ratio (S/I) must be equal or above the acceptable level of $18_{\mathrm{dB}}$. This is required in practice to get appropriate size of cluster to minimize the signal interference between channels using the same frequencies, and to provide user with expected quality of service for communication.

\section{Objective Function:}

In this section, we show how the theoretical foundations of signal-to-co-channel interference ratio (S/I) as described in the previous section is translated to actual formulae and constrains so that problem can be implemented in Excel Solver. Objective function is to:

\section{Minimize value $(\mathbf{S} / \mathbf{I})_{\mathrm{dB}}$,}

subject to the following constraints:

$\mathrm{i}>=0, \mathrm{i}$ is an integer number

$\mathrm{j}>=0, \mathrm{j}$ is an integer number

Minimum value $(\mathrm{S} / \mathrm{I})_{\mathrm{dB}}>=$ Threshold value, and must be positive

The above optimization problem can be represented using the following Excel model:

Cell E9: value of $i$, decision variable to be determined

Cell F9: value of $\mathrm{j}$, decision variable to be determined

Cell G9: value of $\mathrm{N}$ using formula $\mathrm{N}=\mathrm{i} * \mathrm{i}+\mathrm{i} * \mathrm{j}+\mathrm{j} * \mathrm{j}$ using formula (1)

Cell H9: value of frequency reuse ratio using formula $\mathrm{q}=\operatorname{sqrt}\left(3^{*} \mathrm{~N}\right)$ for hexagonal geometry using formula (3)

Cell J9: signal-to-co-channel interference ratio (S/I) using the formula $S / I=1 /\left(2(q-1)^{-k}+2 q^{-k}+2(q+1)^{-k}\right)$ using formula (3)

Cell L9: represents the objective function $\mathrm{s}$

Cell N9: represents the minimum threshold value of $(\mathrm{S} / \mathrm{I}) \mathrm{dB}=18_{\mathrm{dB}}$ in the example.

Constraints on the various cell values are:

Cell E9: integer, and Cell E9 $>=0$

Cell F9: integer, and Cell F9 $>=0$

Cell L9 $>=$ Cell N9, and Cell L9 $>=0$

The next section shows the step by step procedures for enabling Excel Solver, for setting up the objective function, and constraints, and finally executing the solver to find the desired cluster size.

\section{DETERMINING CLUSTER SIZE USING MICROSOFT EXCEL SOLVER}

The following steps show how to formulate objective function and appropriate constraints, that were defined in the previous section, to compute cluster size using Microsoft Excel Solver.

\section{Step 1: Set up the initial Excel Spreadsheet}

- Set columns for initial values of decision variables $i$, and $j$ 
- $\quad$ Set column for Cluster Size N and place formula to calculate N using equation (1)

- Set column for Frequency Reuse Ratio q, and place formula to calculate q using equation (3)

- $\quad$ Set column for (S/I) and place formula to calculate (S/I) using equation (2)

- Set column for $(\mathrm{S} / \mathrm{I}) \mathrm{dB}$ and place formula to calculate $(\mathrm{S} / \mathrm{I}) \mathrm{dB}$ using equation (4).

Figure 3. below shows the sample of initial screen set up

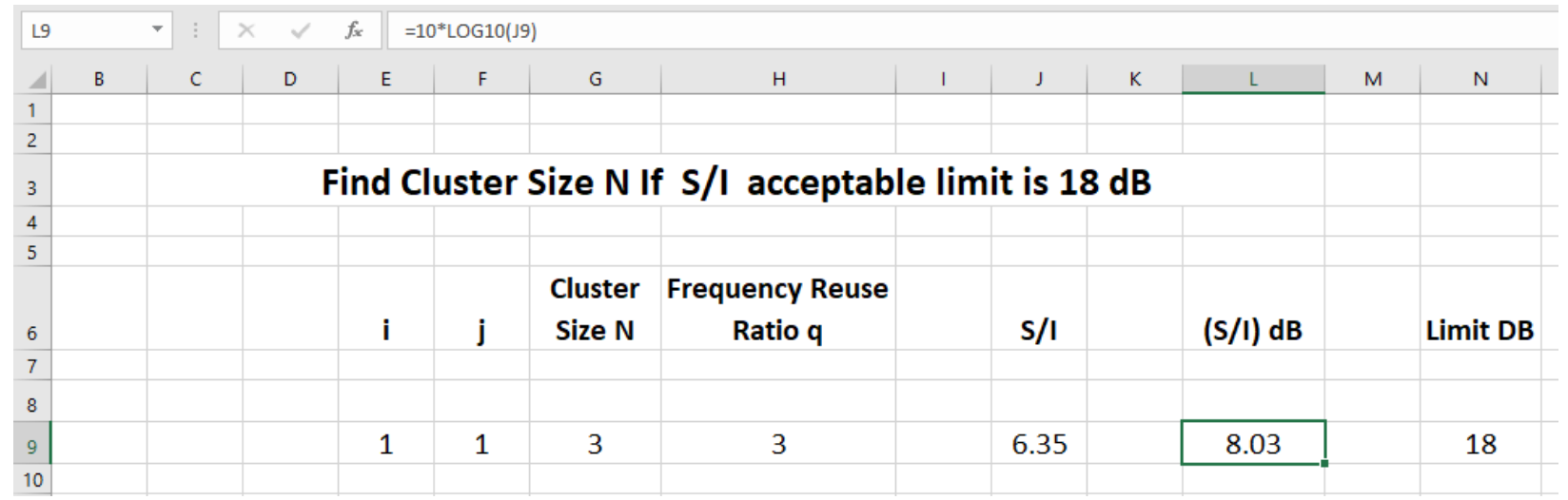

Figure 3. Excel spreadsheet set-up with initial values of $i=1, j=1$.

Step 2: The user has to enable solver by using the following steps:

- Click the File tab, and then click Options below the Excel tab.

- In the Excel Options dialog box, click Add-Ins.

- In the Manage drop-down box, select Excel Add-ins, and then click Go.

- Add-Ins dialog box is displayed in Figure 4.

- In the Add-Ins dialog box of Figure 4, select Solver Add-in, and then click OK. 


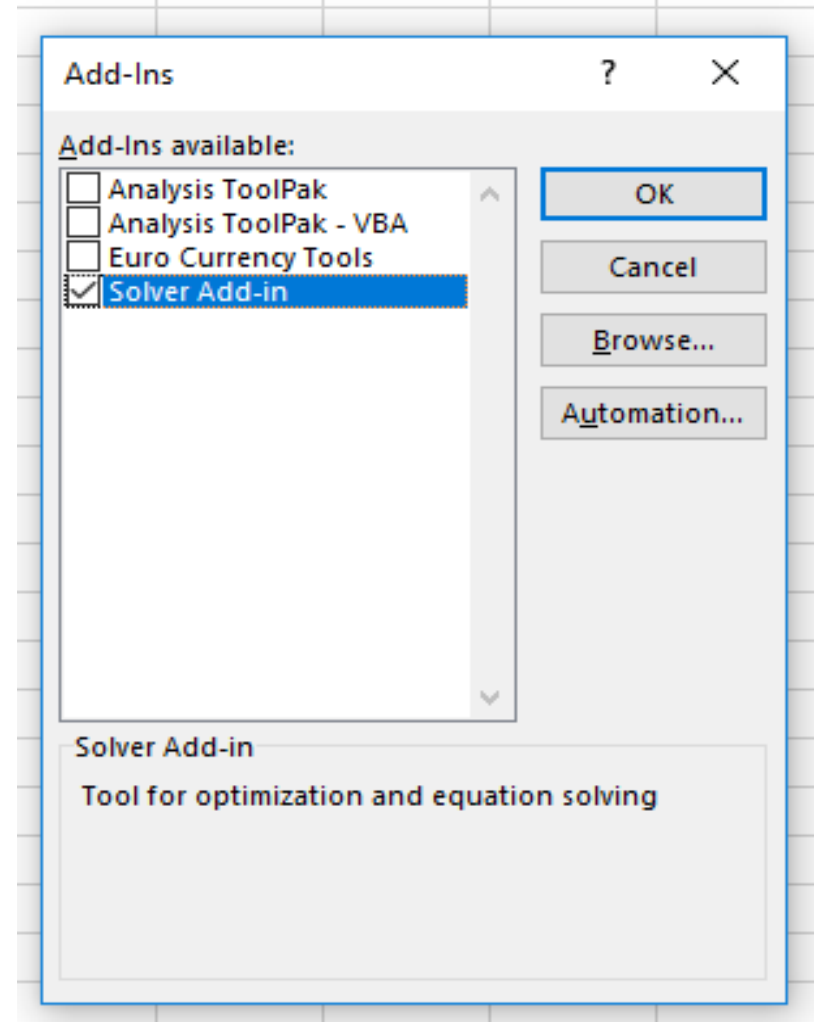

Figure 4. Solver Add-In set up

After the Solver add-in is enabled, Excel will auto-install the Add-in if it is not already installed, and the Solver command will be added to the Analysis group on the Data tab in the ribbon.

Step 3: The user has to define a series of requirements, rules and constraints for EXCEL solver.

Using these requirements, rules and constraints defined in previous section, solver is able to converge to a feasible solution. The Figure 5, and Figure 6 show graphically the set up screens to specify requirements, rules and constraints in Excel Solver. Note \$ symbol below is used to specify absolute location of a cell in Excel.

- In the Set Objective box, enter a cell reference (\$L\$9) for the objective cell that contains the formula.

- Click Min If you want the value of the objective cell to be as small as possible,

- If you want the objective cell to be a certain value, click Value of, and then type the value (18) in the box.

- In the by Changing Variable Cells box, enter reference for each decision variable cell range (\$E\$9: \$F\$9).

- In the Subject to the Constraints box, enter any constraints that you want to apply.

- To accept the constraint and add another, click Add.

- To accept the constraint and return to the Solver Parameters dialog box, click OK.

Figure 5 shows the Excel Solver set up with objective function is set to minimize the value of $(\mathrm{S} / \mathrm{I}) \mathrm{dB}$ subject to the condition that $(\mathrm{S} / \mathrm{I})_{\mathrm{dB}}$ must be greater than $18_{\mathrm{dB}}$. The non-negativity constraints of various variables are also set up in the solver. All unconstrained variables are assumed to be non-negative.

- Select a Solving Method GRG Nonlinear once the solver data is set up. The GRG (Generalized Reduced Gradient method) Nonlinear algorithm is chosen for optimization as shown in Figure 5. The GRG method is commonly used algorithm to solve nonlinear problems in Excel Solver

- Click Solve and to keep the solution values on the worksheet, click Keep Solver Solution, in the SolverResults dialog box. 


\section{Issues in Information Systems}

Volume 21, Issue 1, pp. 37-48, 2020

If Solver has found a solution as shown in Figure 6, the worksheet cells will be changed to show the solution (Figure 7).

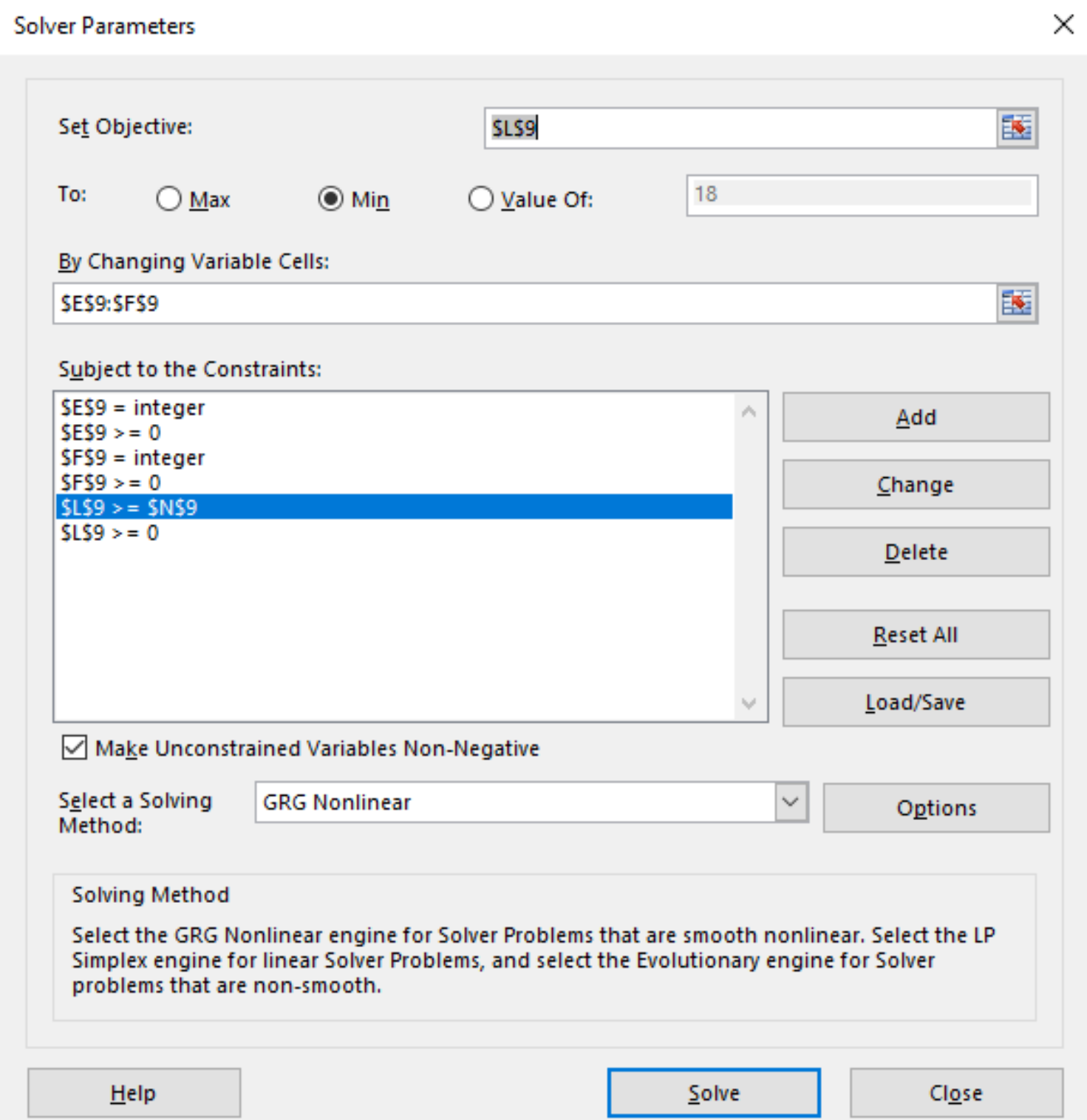

Figure 5. Solver set-up specifying objectives, decision variables and constraints 


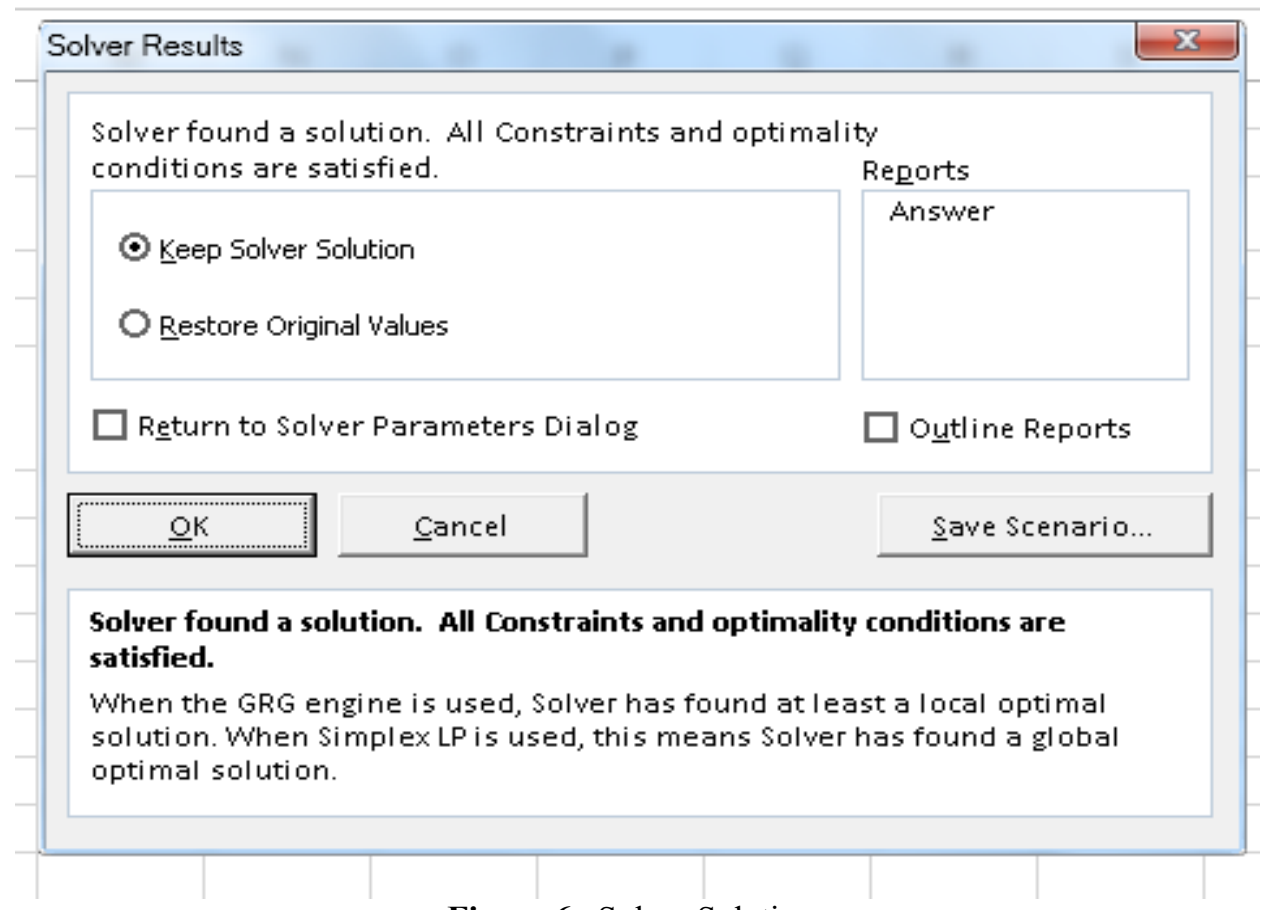

Figure 6. Solver Solutions

\begin{tabular}{|c|c|c|c|c|c|c|c|c|c|c|c|c|c|}
\hline L9 & & $\checkmark \quad:$ & $\checkmark$ & $f_{x}$ & $=10^{*}$ LOG10 & & & & & & & & \\
\hline 4 & B & c & D & E & $\mathrm{F}$ & G & $\mathrm{H}$ & 1 & J & $\mathrm{K}$ & L & M & $\mathrm{N}$ \\
\hline \multirow{2}{*}{\multicolumn{14}{|c|}{$\frac{1}{2}$}} \\
\hline & & & & & & & & & & & & & \\
\hline 3 & & \multicolumn{10}{|c|}{ Find Cluster Size N If $\mathrm{S} / \mathrm{I}$ acceptable limit is $18 \mathrm{~dB}$} & & \\
\hline \multirow{2}{*}{\multicolumn{14}{|c|}{$\frac{4}{5}$}} \\
\hline & & & & & & & & & & & & & \\
\hline 6 & & & & $\mathbf{i}$ & $\mathbf{j}$ & $\begin{array}{l}\text { Cluster } \\
\text { Size N }\end{array}$ & $\begin{array}{c}\text { Frequency Reuse } \\
\text { Ratio q }\end{array}$ & & $S / I$ & & $(S / I) d B$ & & Limit DB \\
\hline 7 & & & & & & & & & & & & & \\
\hline \multicolumn{14}{|l|}{8} \\
\hline 9 & & & & 0 & 3 & 9 & 5 & & 94.77 & & 19.77 & & 18 \\
\hline 10 & & & & & & & & & & & & & \\
\hline
\end{tabular}

Figure 7. Result of final optimal solution from Excel Solver on the spreadsheet

The following Results section discusses in detail the results of executing Excel Solver to find the cluster size.

\section{RESULTS}

The previous section shows the setup of Excel Solver to compute optimal cluster size when the signal-to-co-channel interference is greater than $18_{\mathrm{dB}}$. Figure 3 shows initial values of decision variables $i$, and $j$ which are both set to 1 . As seen from Figure 3 that the calculated value of $(\mathrm{S} / \mathrm{I}) \mathrm{dB}$ is 8.03 which is below the required threshold value of $18_{\mathrm{dB}}$ that is specified in the problem and is hence not optimal.

To get the valid solution, we will invoke Excel Solver. The previous section shows the setup of Excel Solver with objective function, rules and constraints as shown in Figure 4, and Figure 5. When the solver is executed, the result in Figure 6 indicates that an optimised solution is found for the cluster size $\mathrm{N}$ subject to the condition that (S/I) dB must 
be greater than $18_{\mathrm{dB}}$. Figure 7 shows that an optimal solution is found, and cluster size $\mathrm{N}$ is 9 that it meets all specified constraints. With cluster size $\mathrm{N}=9$, the resulting value of $(\mathrm{S} / \mathrm{I})_{\mathrm{dB}}$ is $19.77_{\mathrm{dB}}$ which is greater than $18_{\mathrm{dB}}$ as required. For nonlinear optimization problems Solver cannot guarantee the solution it can find. For a smooth convex nonlinear problem, Solver will normally find the solution, which is globally optimal. But if the problem is non-convex, Solver will normally find only a locally optimal solution, close to the initial values of the decision variables, when the Solver is invoked. To investigate the nature of solutions Solver can find, several different initial values of decision variables are used and solutions are examined. Figure 8 shows the set up of spreadsheet with different initial values of decision variables $i=1, j=2$ from that of Figure 3 and the initial computations is shown.

\begin{tabular}{|c|c|c|c|c|c|c|c|c|c|c|c|c|c|}
\hline$F 12$ & & 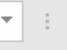 & $\times \checkmark$ & $f_{x}$ & & & & & & & & & \\
\hline 4 & B & c & D & E & $\mathrm{F}$ & G & $\mathrm{H}$ & 1 & J & K & L & M & $\mathrm{N}$ \\
\hline \multirow{2}{*}{\multicolumn{14}{|c|}{$\begin{array}{l}1 \\
2\end{array}$}} \\
\hline & & & & & & & & & & & & & \\
\hline 3 & & \multicolumn{10}{|c|}{ Find Cluster Size N If $\mathrm{S} / \mathrm{I}$ acceptable limit is $18 \mathrm{~dB}$} & & \\
\hline \multirow{2}{*}{\multicolumn{14}{|c|}{$\frac{4}{5}$}} \\
\hline & & & & & & & & & & & & & \\
\hline 6 & & & & i & $\mathbf{j}$ & $\begin{array}{l}\text { Cluster } \\
\text { Size N }\end{array}$ & $\begin{array}{c}\text { Frequency Reuse } \\
\text { Ratio q }\end{array}$ & & $s / I$ & & $(\mathrm{~s} / \mathrm{l}) \mathrm{dB}$ & & Limit DB \\
\hline 7 & & & & & & & & & & & & & \\
\hline \multicolumn{14}{|l|}{8} \\
\hline 9 & & & & 1 & 2 & 7 & 5 & & 53.38 & & 17.27 & & 18 \\
\hline 10 & & & & & & & & & & & & & \\
\hline
\end{tabular}

Figure 8. Excel spreadsheet set-up with different initial values of $i=1, j=2$

As seen from Figure 8 with initial values of $i=1, j=2$, the calculated signal-to-co-channel intereference ratio is $17.27_{\mathrm{dB}}$ which is below the accepted ratio of $18_{\mathrm{dB}}$. When Excel Solver is executed with these values of decision variables, the optimal solution is found, and displayed in Figure 9. As can be seen from Figure 9, the Solver gave the same value of the cluster size $\mathrm{N}=9$ as in Figure 7 when different initial values are used, and signal-to-co-channel intereference ratio is $19.77_{\mathrm{dB}}$, which is above the minimum accepted ratio of $18_{\mathrm{dB}}$.

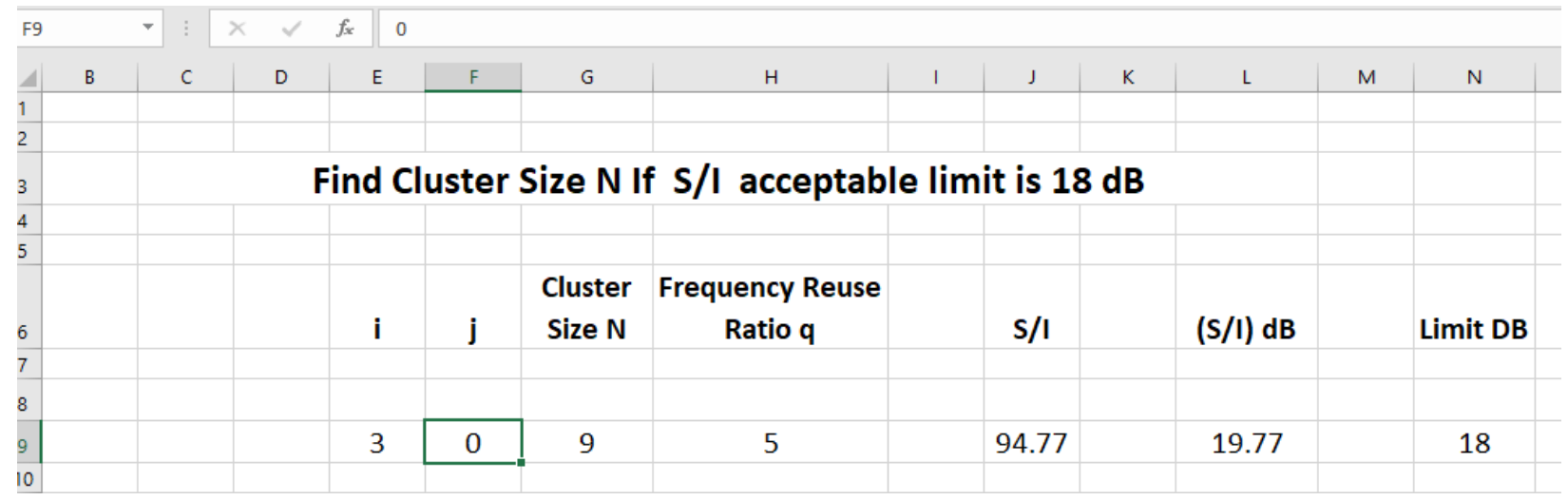

Figure 9. Result of final optimal solution from Excel Solver on the spreadsheet

Table 2 shows the results with different initial values of $i, j$ before and after the Solver is executed. In all cases, Solver when executed with different values of $i, j$ converged to the same cluster size $\mathrm{N}=9$ to meet the requiirements that minimum $\mathrm{S} / \mathrm{I}$ dB must exceed $18_{\mathrm{dB}}$.

Table 3 shows the results with different initial values of $i, j$ before and after the Solver is executed when threshold limit is changed from 18 dB to $21 \mathbf{d B}$. In all cases, Solver when executed with different values of $i, j$ converged to the same cluster size $\mathrm{N}=12$ to meet the requiirements that minimum $\mathrm{S} / \mathrm{I} \mathrm{dB}$ must exceed $21_{\mathrm{dB}}$.

As can be seen by comparing Table 2 and Table 3 when threshold limit is raised from 18 dB to 21 dB, cluster size has increased from 9 to 12 . This is expected as the signal-to-co-channel intereference ratio is increased system will be able 
to accommodate more coverage area using the allocated frequency channels. In other words we can add three additional cells in the coverage area of the cluster than before. Also the value of frequency reuse ratio $\mathrm{q}$ also increased from 5 to 6 .

Table 2. Results of $N, q, S / I_{d B}$ before and after execution of Solver with different values of $i$, and $j$

\begin{tabular}{|c|c|c|c|c|c|c|c|c|c|}
\hline \multicolumn{10}{|c|}{ Threshold Limit: 18dB } \\
\hline \multicolumn{5}{|c|}{ Before Solver is executed } & \multirow{2}{*}{\multicolumn{5}{|c|}{$\begin{array}{c}\text { After Solver is executed } \\
\text { Final Values after Solver is executed }\end{array}$}} \\
\hline Ini & lues & & ted & & & & & & \\
\hline $\mathbf{i}$ & $\mathbf{j}$ & $\mathbf{N}$ & $\mathbf{q}$ & $\mathrm{S} / \mathbf{I}_{\mathrm{dB}}$ & $\mathbf{i}$ & $\mathbf{j}$ & $\mathbf{N}$ & $\mathbf{q}$ & $\mathrm{S} / \mathbf{I} \mathrm{dB}$ \\
\hline 0 & 1 & 1 & 2 & -8.59 & $\mathbf{0}$ & 3 & 9 & 5 & 19.77 \\
\hline 1 & 1 & 3 & 3 & 8.03 & $\mathbf{0}$ & 3 & 9 & 5 & 19.77 \\
\hline 0 & 2 & 4 & 3 & 11.36 & $\mathbf{0}$ & 3 & 9 & 5 & 19.77 \\
\hline 1 & 2 & 7 & 5 & 17.27 & 3 & 0 & 9 & 5 & 19.77 \\
\hline 2 & 2 & 12 & 6 & 22.54 & $\mathbf{0}$ & 3 & 9 & 5 & 19.77 \\
\hline 0 & 3 & 9 & 5 & 19.77 & $\mathbf{0}$ & 3 & 9 & 5 & 19.77 \\
\hline 4 & 3 & 37 & 11 & 32.86 & 3 & $\mathbf{0}$ & 9 & 5 & 19.77 \\
\hline
\end{tabular}

Table 3. Results of N, q, S/I $\mathrm{dB}$ before and after execution of Solver with different values of $\mathrm{i}$, and $\mathrm{j}$

Threshold Limit: $21_{\mathrm{dB}}$

\begin{tabular}{|c|c|c|c|c|c|c|c|c|c|}
\hline \multicolumn{5}{|c|}{ Before Solver is executed } & \multirow{2}{*}{\multicolumn{5}{|c|}{$\begin{array}{c}\text { After Solver is executed } \\
\text { Final Values after Solver is executed }\end{array}$}} \\
\hline Init & lues & & & ues & & & & & \\
\hline $\mathbf{i}$ & $\mathbf{j}$ & $\mathbf{N}$ & $q$ & $\mathbf{S} / \mathbf{I} \mathrm{dB}$ & $\mathbf{i}$ & $\mathbf{j}$ & $\mathbf{N}$ & $\mathbf{q}$ & $\mathrm{S} / \mathbf{I}_{\mathrm{dB}}$ \\
\hline 0 & 1 & 1 & 2 & -8.59 & 2 & 2 & 12 & 6 & 22.54 \\
\hline 1 & 1 & 3 & 3 & 8.03 & 2 & 2 & 12 & 6 & 22.54 \\
\hline 0 & 2 & 4 & 3 & 11.36 & 2 & 2 & 12 & 6 & 22.54 \\
\hline 1 & 2 & 7 & 5 & 17.27 & 2 & 2 & 12 & 6 & 22.54 \\
\hline 2 & 2 & 12 & 6 & 22.54 & 2 & 2 & 12 & 6 & 22.54 \\
\hline 0 & 3 & 9 & 5 & 19.77 & 2 & 2 & 12 & 6 & 22.54 \\
\hline 4 & 3 & 37 & 11 & 32.86 & 2 & 2 & 12 & 6 & 22.54 \\
\hline
\end{tabular}

\section{LIMITATIONS OF EXCEL SOLVER}

It is worthwhile to keep in mind that the Excel Solver may not come up with correct solution in every situation (Evans, 2008). The Solver works if the number of decision variables is within a certain upper limit (about 200 decision variables). There are also upper limits on number of constraints. The constraint limit varies with types of algorithm (linear, nonlinear) used for optimization and the form of the constraints. These limitations do not affect our computation as number of decision variables are only two in our examples, and constraints are very simple. However, 
if error occurs, it is suggested that the Solver be re-invoked with new values of decision variables. Adding reasonably tight upper and lower bound constraints on variables may also speed up the Solver to reach a correct solution.

\section{CONCLUSIONS}

Excel Solver techniques is widely used in business applications because of its ease of use. The author selected one specific problem in Cellular technology as a test case to examine the usefulness of Excel as a tool for complex engineering problem. The reason for selecting this application is that the theory of Cellular technology is based on complex mathematical models. The resultant output of these models cannot be analyzed with easily available software tools. With this purpose in mind, the author wants to explore commonly available tools that will enable one to analyze cellular technology concepts without spending inordinate amount of efforts to get a mathematical solution. Many of the engineering computational problems are traditionally solved using programs like MATLAB or other simulation software. These software tools may provide higher performance and flexibility but has stiff learning curve for users of these software. To demonstrate the usefulness of such tool, the author decided to handle one specific problem in Cellular technology - "Determine the optimal cluster size of cell configurations that will meet signal-to-co-channel interference threshold". The algorithm to determine cluster size is adapted for implementation in Microsoft Excel. The Microsoft Excel Solver feature in Excel is specifically suited for solving optimization problems and is used to determine cluster size in a cellular network. The author has demonstrated that solution to find cluster size can easily be computed using Microsoft Excel Solver and one can experiment with different scenarios to study the effect of modifying parameters on the size of cell cluster as shown in the paper. As a part of further experimentation, author is reviewing other examples from wireless network which can easily be modeled and implemented using simple Excel techniques. The author has used this tool in classroom to convey the subject matter concepts, and to demonstrate how to find solutions to complex optimization problem using simple tools such as Microsoft Excel.

\section{REFERENCES}

Adediran, Y.A., Lasisi ,H., \& Okedere O.B.) (2017). Interference management techniques in cellular networks: A review. Available: https://www.cogentoa.com/article/10.1080/23311916.2017.1294133

Chandrakantha, L. (2011). Using Excel Solver in Optimization Problems. Proceedings of the Twenty-third Annual International Conference on Technology in Collegiate Mathematics, Denver, Colorado, March 17-20, Available: http://archives.math.utk.edu/ICTCM/i/23/C006.html

Waterloo (2012). Fundamentals of Cellular Communications and Multiple Access Techniques, Available: https://ece.uwaterloo.ca/ ece414/ece414_chapter5_w12.pdf

Cox, D.D. (1982). Co-channel Interference Considerations in Frequency Reuse Small Coverage Area Radio System. IEEE Trans. Commun. 30.

Diakoku K., Ohdate H. (1983). Optimal Channel Reuse in Cellular Land Mobile Radio System. IEEE Trans. Veh. Tech, VT-32.

El Jaafreh, Y. G. (2000). Co-channel and Adjacent Channel Interference Calculations in Cellular. Communications Systems, Journal of King Saud University - Engineering Sciences, Volume 12, Issue 1.

Evans, J. R. (2008). Teaching Note-Some Practical Issues with Excel Solver: Lessons for Students and Instructor. INFORMS Transactions on Education 8(2):89-95. Available: https://pubsonline.informs.org/doi/pdf/10.1287/ited.1070.0006

Mark, J. W., Zhuang, W. (2003). Wireless Communications and Networking. Pearson

Safak A., Prasad R. (1991). Effects of Correlated Shadowing Signals On Channel Re-use in Mobile Radio System. IEEE Trans, on Veh. Tech, 40 (4).

Stallings, W., Beard, C. (2016). Wireless Communication Networks and Systems, Pearson Higher Education.

Stubert, G.L. (2017). Principles of Mobile Communication, $4^{\text {th }}$ Edition, Springer.

Winston, W. (2014). Marketing Analytics: Data-Driven Techniques with Microsoft Excel, Wiley.

Winston, W. (2014). Microsoft Excel 2013 Data Analysis and Business Modeling, Microsoft Press. 